\title{
Évaluation comparée du risque chimique et du risque radiologique pour l'environnement
}

\author{
K. BEAUGELIN-SEILLER ${ }^{1}$, J. GARNIER-LAPLACE ${ }^{2}$
}

(Manuscrit reçu le 12 avril 2007, accepté le 30 juillet 2007)

RÉSUMÉ Protéger l'environnement est aujourd'hui une problématique globale qui concerne toutes les activités de l'homme. Il s'agit donc d'être capable de démontrer la compatibilité de ces activités avec la protection des milieux naturels, mais aussi si nécessaire d'agir pour pallier à un risque potentiel ou avéré, et ce généralement dans le cadre d'une réglementation de plus en plus stricte. Dans le domaine chimique, la prise en compte de cette problématique se traduit par l'existence de méthodes dédiées à l'évaluation du risque environnemental. Dans le domaine radiologique, la nécessité de disposer de tels outils s'est accentuée récemment, afin de pouvoir justifier aux yeux du public et en toute transparence le rejet de substances radioactives dans l'environnement, de répondre aux exigences des nouvelles réglementations en matière de protection de l'environnement et enfin d'harmoniser les approches entre radioprotection humaine et radioprotection de l'environnement, mais aussi entre polluants chimiques et radioactifs, simultanément présents par exemple dans les rejets de routine des centres nucléaires de production d'électricité. Dans cette optique d'harmonisation, les démarches d'évaluation du risque à l'environnement lié à la présence de radionucléides ou de substances chimiques sont présentées depuis les concepts théoriques jusqu’à leur mise en œuvre comparative, approche nouvelle en cours de développement dont les premiers résultats sont présentés.

ABSTRACT Compared assessment of chemical risk and radiological risk for the environment.

The environmental protection is today a global issue that concerns all the human activities. The challenge is to be able to prove their compatibility with the protection of the natural environment, but also if required to manage adequately a potential or established risk, generally in the context of ever strict regulations. Regarding the chemical aspects, known methods are available for the assessment of the associated risk for the environment. From the radiological point of view, the necessity to have such tools recently became more apparent, in order to justify for the public and with a full transparency the release of radioactive substances into the environment, to meet the requirements of the new regulations in terms of environmental protection and finally to harmonize the approaches between human and environmental radioprotection as well as between chemical and radioactive pollutants, simultaneously present for example in routine releases from nuclear power plants. At this end of harmonization, the assessment methodologies of the environmental risk linked to radionuclides or chemical substances are exposed from the theory to their comparative application, a new approach under development from which the first results are summarised.

Keywords: radionuclides / chemicals / risk / assessment / comparison

1 IRSN/DEI/SECRE, Laboratoire de Modélisation de l'Environnement, bâtiment 159, Centre de Cadarache, B.P. 3, 13115 St Paul lez Durance, France.

2 IRSN, Laboratoire de Radioécologie et d'Écotoxicologie, Centre de Cadarache, B.P. 3, 13115 St Paul lez Durance, France. 


\section{Introduction}

La protection de l'homme et de l'environnement vis-à-vis des rayonnements ionisants et des autres polluants a fait l'objet d'une séparation historique à la fois sur le plan de la $R \& D$ et sur le plan réglementaire. L'essor industriel du domaine nucléaire s'est accompagné du besoin impératif d'un système de radioprotection pour les travailleurs. Et c'est à partir de ce système que s'est bâti par extrapolation celui de la radioprotection du public, bien que la voie d'exposition majeure de celuici soit l'ingestion chronique (versus inhalation ou blessure en exposition aiguë). Il a alors été posé comme postulat que, si l'homme était protégé, alors les autres espèces vivantes moins radiosensibles l'étaient aussi (ICRP, 1991). Ce postulat a prévalu jusqu'à la fin des années 90 , où s'est fait jour une préoccupation croissante quant à la protection de l'environnement per se ; disposer de méthodes et d'outils prouvant explicitement la protection de l'environnement vis-à-vis des rayonnements ionisants est devenu une nécessité. Conjointement, les objectifs de la protection de l'environnement se sont élargis au contexte des pollutions multiples dans une optique de développement durable. C'est ainsi que les principes de l'évaluation du risque pour l'environnement, déjà appliqués aux produits chimiques, sont apparus de façon consensuelle comme pouvant également s'appliquer aux substances radioactives, en permettant de démontrer l'atteinte d'un niveau approprié de protection des écosystèmes et en répondant à l'absence de méthode pour caractériser le risque radiologique pour l'environnement (ERICA, 2007).

Il n'existe pas d'argumentation scientifique fondée pour justifier de considérer les radionucléides autrement que les polluants chimiques vis-à-vis de l'évaluation $\mathrm{du}$ risque qu'ils induisent pour l'environnement. Cependant, il existe quelques différences conceptuelles majeures telles que l'expression des effets liés à une exposition à des radionucléides rapportée à l'énergie déposée dans l'organisme et non à la concentration d'exposition dans le milieu. Malgré ces différences, l'approche ERA (Environmental Risk Assessment) traditionnelle peut être appliquée à la fois pour des substances chimiques et pour des substances radioactives. Toutefois, cette méthode est dédiée à une évaluation conservative du risque, substance par substance. Cette analyse mono-polluant n'autorise pas une évaluation relative des risques liés à des stresseurs de nature différente (Pennington et al., 2004). C'est pourquoi une nouvelle approche est proposée, basée sur la notion de pourcentage d'espèces affectées (PAF - Potentially Affected Fraction -, fraction potentiellement affectée des espèces présentes). Déjà mise en œuvre pour les polluants chimiques dans le cadre des développements liés aux analyses de cycle de vie (ACV, Pennington et al., 2006), elle est en cours d'adaptation à l'IRSN pour y inclure les radionucléides, et un premier cas d'application a été réalisé sur les rejets de routine d'un centre nucléaire de production d'électricité. 


\section{La méthode européenne classique d'évaluation du risque pour l'environnement}

Ce paragraphe n'a pas pour objectif de présenter de façon exhaustive les tenants et les aboutissants de la méthode et de son environnement contextuel, précédemment décrits (IRSN, 2006). Il vise simplement à rappeler les éléments de base nécessaires à sa compréhension et à l'explication de la mise en œuvre comparée de l'évaluation du risque radiologique et chimique.

\subsection{Principes généraux communs}

En Europe, la démonstration de l'occurrence d'un risque avéré ou potentiel pour l'environnement en lien avec la présence ou le rejet d'un polluant dans un écosystème donné est pour les polluants chimiques (EC, 2003) traditionnellement basée sur une méthode en quatre phases (Fig. 1) adoptées également aujourd'hui pour les radionucléides (ERICA, 2007):

- l'identification des dangers, par un inventaire qualitatif et quantitatif des substances à étudier ;

- l'analyse des effets, reposant sur l'examen critique des relations exposition-réponse associées à chaque substance pour déterminer un niveau de référence prévu sans effet (PNEC, Predicted No Effect Concentration pour les polluants chimiques; PNEDR, Predicted No Effect Dose Rate pour les radionucléides);

- l'analyse des expositions par la mesure ou le calcul des niveaux d'exposition des différents compartiments d'intérêt dans l'écosystème (PEC, Predicted Environmental Concentration pour les polluants chimiques, PEDR, Predicted Environmental Dose Rate pour les radionucléides) ;

- la caractérisation du risque, par confrontation des résultats des phases d'analyse des effets (PNEC et PNEDR) et de l'exposition (PEC et PEDR).

Ces quatre phases sont généralement exécutées de manière séquentielle, selon une approche graduée en trois étapes (Suter et al., 2000). La première correspond à un screening qui permet, à partir de méthodes simples et conservatives, d'identifier parmi les situations et les contaminants ceux présentant un risque écologique potentiel. La deuxième est une étape intermédiaire utilisant des données génériques appliquées seulement aux situations et polluants d'intérêt, étape souvent associée à une estimation déterministe du risque. La dernière étape, la plus raffinée, fait appel à des données site-spécifiques, auxquelles est associée une évaluation de leur incertitude, conjuguée à une estimation probabiliste du risque. L'enchaînement de ces trois étapes entraîne donc un gain qualitatif et quantitatif en termes de données (Fig. 2), générant une augmentation de la représentativité mais aussi des ressources mises en œuvre. 


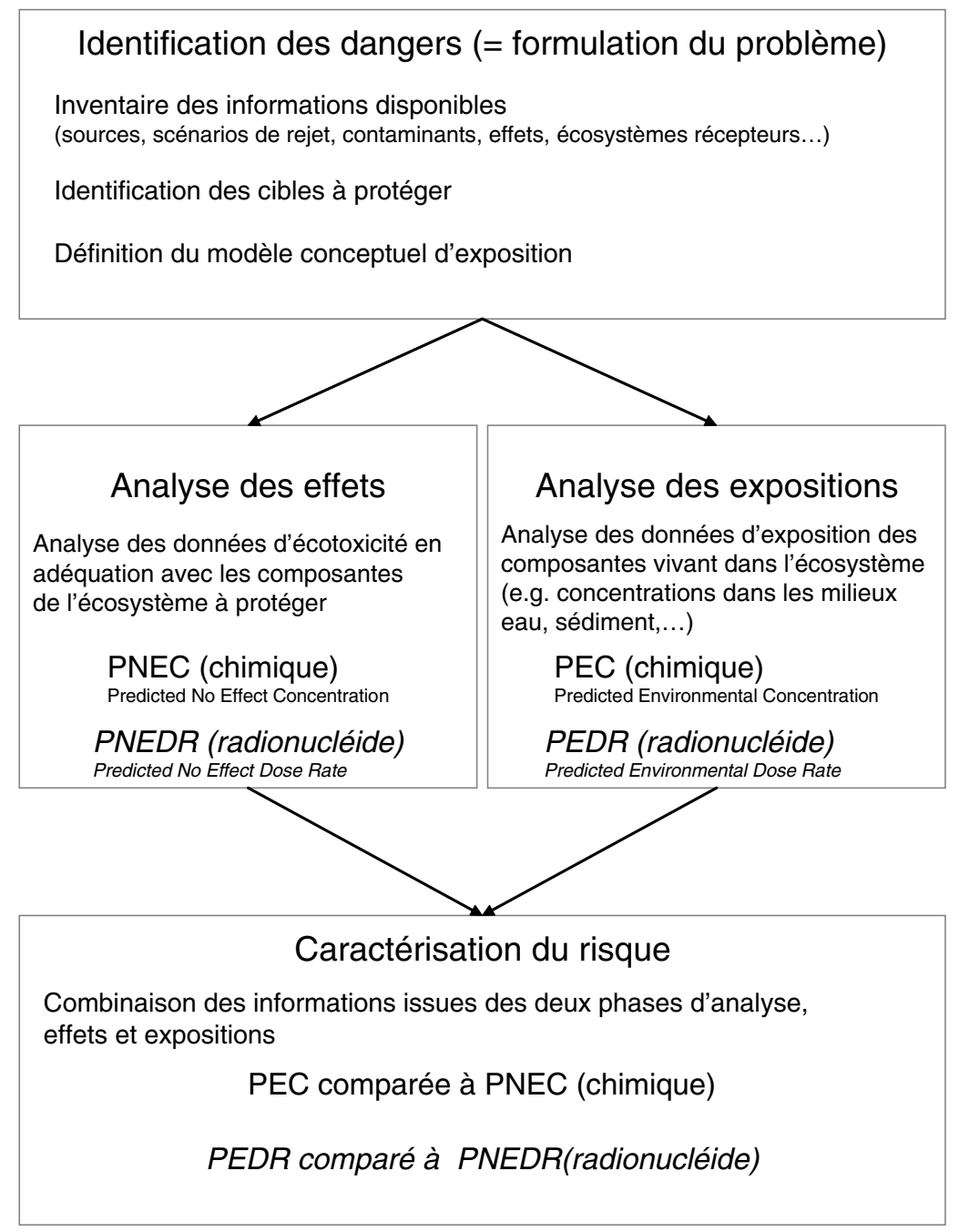

Figure 1 - Les quatre étapes basiques de l'évaluation du risque pour l'environnement.

The four basic steps of the environmental risk assessment method.

\subsection{Formulation du problème}

Six critères discriminant les différentes méthodes existantes d'évaluation du risque écologique caractérisent la formulation du problème : le domaine temporel visé (évaluation prospective, rétrospective, les deux); les objectifs de l'évaluation 


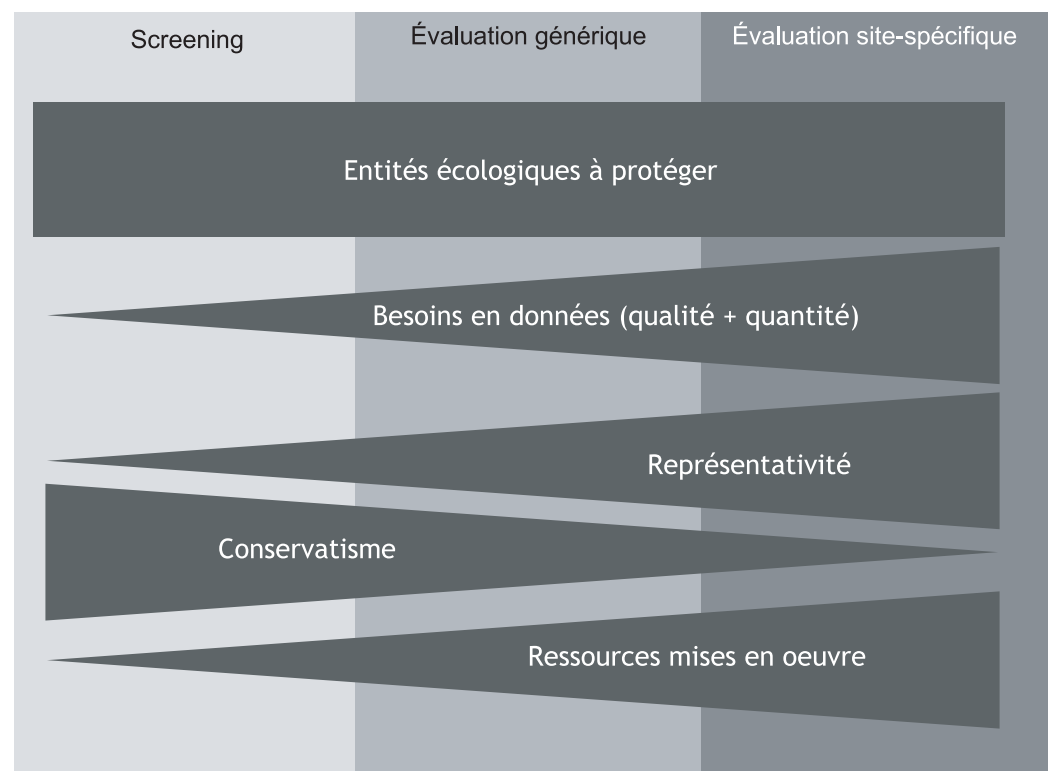

Figure 2 - Évolution des besoins et de la représentativité des étapes successives d'une évaluation graduée des risques.

Requirement and representativeness evolution through the successive steps of a graded risk assessment.

(établissement de normes, de seuils de protection, d'indices de risques, ...) ; le niveau écologique de protection visé (individu, population, écosystème) ; l'articulation des étapes (screening, étude générique ou site-spécifique); la définition éventuelle du niveau de risque acceptable; la caractérisation de l'exposition (chronique, aiguë).

\subsection{Analyse des expositions}

L'objectif de l'analyse des expositions est de fournir par la mesure ou la modélisation un élément de comparaison avec les résultats de la phase d'analyse des effets, PEC pour les substances chimiques, PEDR pour les radionucléides. En effet, l'une des spécificités des radionucléides réside dans la relation expositiondose-effet, les données d'effet étant rapportées à la dose absorbée, quantité de base utilisée pour exprimer le niveau d'exposition de tout organisme aux rayonnements ionisants, exprimée en Gy. Cette grandeur, employée pour caractériser l'exposition aiguë, est rapportée à la durée d'exposition en situation chronique, et devient ainsi un débit de dose, exprimé en Gy par unité de temps. Ces concepts de 
dose et de débit de dose ne suffisent pas à eux seuls à caractériser l'effet des rayonnements ionisants, ils doivent être considérés en lien avec les différentes voies d'exposition possibles de l'organisme, à savoir l'irradiation externe au contact ou à distance, autre spécificité des radionucléides, et la contamination (irradiation interne).

Sous l'hypothèse de l'additivité entre voies d'exposition et entre radionucléides, la détermination du débit de dose d'exposition, PEDR, se fait alors sur la base du jeu d'équations (1) à (5).

$\operatorname{PEDR}(r n$, organisme $)=P E D R_{\text {ext }}(r n$, organisme $)+P E D R_{\text {int }}(r n$, organisme $)$

avec

$P E D R_{\text {ext }}(r n$, organisme) débit de dose prévisible dans l'environnement par exposition externe de l'organisme au radionucléide $r n$,

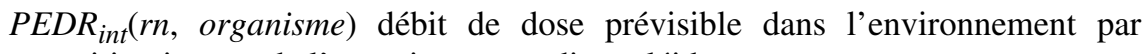
exposition interne de l'organisme au radionucléide $r n$,

$P E D R_{\text {ext }}($ rn, organisme $)=F O($ organisme, milieu $)$

$$
\times D C C_{e x t}(r n, \text { organisme }) \times C(r n, \text { milieu })
$$

avec

FO(organisme, milieu) facteur d'occupation du milieu d'exposition par l'organisme,

$D C C_{\text {ext }}(r n$, organisme $)$ coefficient de conversion de dose en exposition externe de l'organisme pour le radionucléide $r n$,

$C(r n$, milieu $)$ concentration du radionucléide $r n$ dans le milieu d'exposition,

$$
\begin{aligned}
P E D R_{\text {int }}(r n, \text { organisme })= & B C F(r n, \text { organisme }, \text { milieu }) \\
& \times C(r n, \text { milieu }) \times D C C_{\text {int }}(r n, \text { organisme })
\end{aligned}
$$

avec

$B C F(r n$, organisme, milieu) facteur de transfert/concentration du radionucléide $r n$ par l'organisme à partir du milieu,

$D C C_{\text {int }}(r n$, organisme $)$ coefficient de conversion de dose en exposition interne de l'organisme pour le radionucléide $r n$, 


$$
\begin{aligned}
& \operatorname{PEDR}(\text { rn }, \text { organisme })= \\
& {\left[\begin{array}{c}
F O(\text { organisme }, \text { milieu }) \times D C C_{\text {ext }}(r n, \text { organisme }) \\
+B C F(\text { rn }, \text { organisme }, \text { milieu }) \times D C C_{\text {int }}(r n, \text { organisme })
\end{array}\right] \times C(r n, \text { milieu })} \\
& P E D R(\text { organisme })=\sum_{r n} \operatorname{PEDR}(r n, \text { organisme }) .
\end{aligned}
$$

Le calcul dosimétrique nécessite donc la détermination des coefficients de conversion de dose, ou DCC. Celle-ci repose sur la notion d'organismes de référence ${ }^{1}$, espèces modèles représentatives de l'ensemble de l'écosystème cible, dans sa structure et son fonctionnement. Fixer la géométrie, les habitats et le mode de vie de ces espèces permet alors le calcul physique des coefficients de dose associés à chaque combinaison (radionucléide, organisme, voie d'exposition). Différentes méthodes sont applicables, de l'application de formules analytiques à la mise en œuvre de codes de transport physique. L'IRSN a choisi une solution intermédiaire, en développant un outil informatique dédié, EDEN (BeaugelinSeiller et al., 2006), qui autorise de façon simple et conviviale la détermination de ces coefficients pour tout radionucléide, tout organisme et tout scénario d'exposition définis par l'utilisateur.

La détermination des concentrations dans les différents compartiments peut être basée soit sur la mesure, à privilégier dans la mesure du possible, ou par défaut sur la modélisation, en appliquant des modèles de transport et de transfert. Dans ce domaine, l'IRSN est en train de développer une plate-forme de modélisation multimédia, accueillant modèles conceptuels et bases de données associées (Gonze et al., 2004).

Enfin, les propriétés de décroissance physique des radionucléides imposent de prendre en compte dans les phases d'analyse des expositions et d'effets les radionucléides fils ainsi produits.

\subsection{Analyse des effets}

Deux méthodes sont appliquées au plan international pour définir les valeurs de référence dites sans effet (PNEC et PNEDR), en fonction de l'abondance des données d'écotoxicité de base nécessaires.

\footnotetext{
1 Par analogie à l'homme de référence, espèces sélectionnées pour représenter l'ensemble de la faune et de la flore, et caractérisées en termes de géométrie, composition chimique élémentaire, et modes de vie afin de définir une scène d'exposition écologiquement plausible pour procéder au calcul dosimétrique.
} 
TABLEAU I

Nature des données d'écotoxicité de base nécessaires à l'établissement des valeurs de référence sans effet, en fonction du type d'exposition et de contaminant.

Nature of ecotoxicity data required to establish no effect reference values, depending on the kind of exposure and contaminant.

\begin{tabular}{|c|c|c|c|}
\hline Exposition & Données disponibles & Facteur de sécurité & Extrapolation \\
\hline \multirow[t]{5}{*}{ Chronique } & $\begin{array}{l}\text { Au moins une } \mathrm{CL}(\mathrm{E})_{50} \mathrm{a} / \mathrm{DE}_{50} \text { d'un essai court } \\
\text { terme pour chacun des } 3 \text { niveaux trophiques } \\
\text { standards (poissons, daphnies et algues) }\end{array}$ & 1000 & \multirow[t]{7}{*}{$\begin{array}{l}\text { - Aigu vs. chronique } \\
\text { - D'une espèce unique } \\
\text { à l'écosystème }\end{array}$} \\
\hline & $\begin{array}{l}\text { Un(e) } \mathrm{NOEC}^{\mathrm{b}} / \mathrm{DDE}_{10} \text { d'un essai à long terme } \\
\text { (poissons ou daphnies) }\end{array}$ & 100 & \\
\hline & $\begin{array}{l}\text { Deux NOEC / } \mathrm{DDE}_{10} \text { d'essais à long terme pour } \\
3 \text { niveaux trophiques (poissons et/ou daphnies } \\
\text { et/ou algues) }\end{array}$ & 50 & \\
\hline & $\begin{array}{l}\text { Trois NOEC / } \mathrm{DDE}_{10} \text { d'essais à long terme pour } \\
3 \text { niveaux trophiques (poissons, daphnies et } \\
\text { algues le plus souvent) }\end{array}$ & 10 & \\
\hline & $\begin{array}{l}\text { Au moins } 10 \mathrm{NOEC} / \mathrm{DDE}_{10} \text { sur } 8 \text { espèces : } \\
\text { méthode des SSD }\end{array}$ & 1 à 5 (au cas par cas) & \\
\hline \multirow[t]{2}{*}{ Aigu } & $\begin{array}{l}\text { Au moins une } \mathrm{CL}(\mathrm{E})_{50} / \mathrm{DE}_{50} \text { d'un essai court } \\
\text { terme pour chacun des } 3 \text { niveaux trophiques } \\
\text { standards (poissons, daphnies et algues) }\end{array}$ & 100 & \\
\hline & $\begin{array}{l}\text { Au moins } 10 \mathrm{CL}(\mathrm{E})_{50} / \mathrm{DE}_{50} \text { sur } 8 \text { espèces : } \\
\text { méthode des } \mathrm{SSD}\end{array}$ & 1 à 5 (au cas par cas) & \\
\hline
\end{tabular}

${ }^{a}$ Concentration d'une substance qui cause une variation de $x \%$ sur le critère d'effet étudié chez des individus exposés pendant une durée donnée, par rapport au groupe témoin (non exposé). La même définition s'applique à la Dose $(\mathrm{DE} x \%)$ ou au débit de dose (DDEx \%). Ces paramètres sont estimés par modélisation de la relation (concentration-effet), (dose-effet), (débit de dose-effet).

b No-Observed-Effect Concentration/Dose/Dose Rate. Lors de l'établissement de relations concentration-effets, la NOEC est la plus forte concentration testée ne produisant pas un effet statistiquement significatif par rapport au groupe témoin, non exposé. Cette définition s'applique également aux relations dose-effet et débit de dose-effet.

La première méthode, dite des facteurs d'extrapolation ou de sécurité, est employée lorsque les données requises sont peu nombreuses. Elle consiste à appliquer à la plus faible des valeurs d'écotoxicité retenues un facteur de sécurité allant de 1 à 1000, selon la nature, la quantité et la qualité des données disponibles (Tab. I).

La seconde méthode est celle dite des SSD (Species Sensitivity Distribution, Posthuma et al., 2002), recommandée dès lors que le nombre des données d'écotoxicité de base est suffisant pour une exploitation statistique (EC, 2003). Elle consiste à décrire la variation de sensibilité observée dans un ensemble d'espèces considéré comme représentatif d'une communauté ou d'un écosystème, au moyen de sa courbe de fréquence cumulée en fonction des conditions d'exposition. Cette courbe est obtenue par ajustement d'une loi statistique 


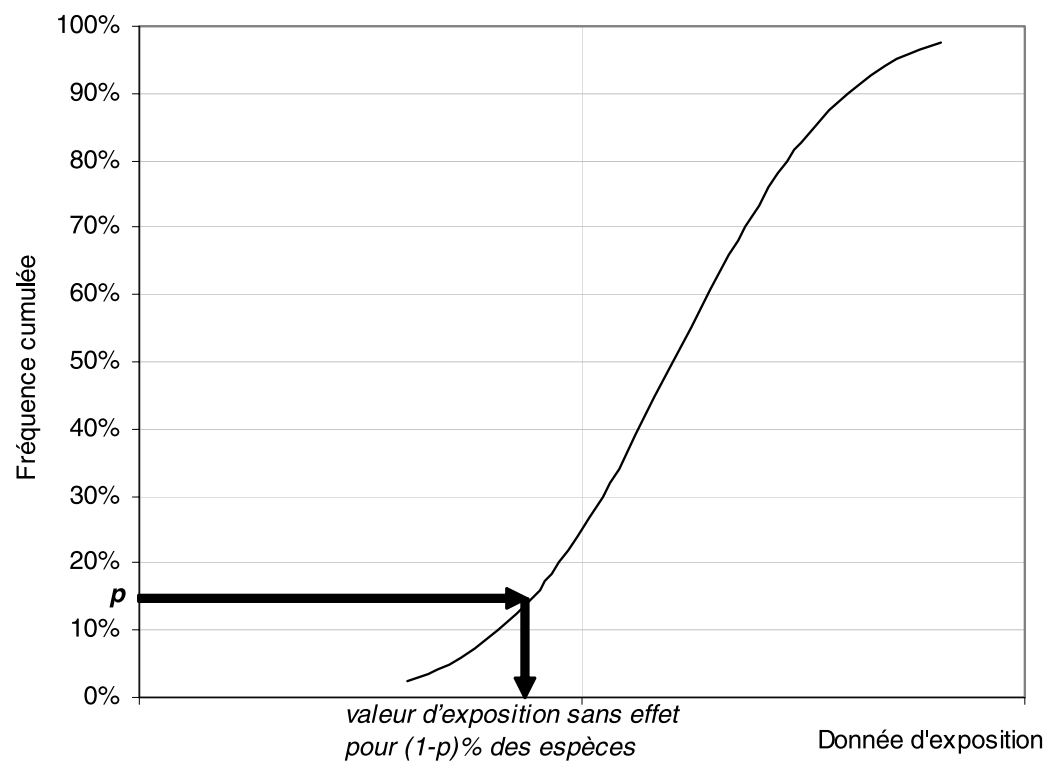

Figure 3 - Utilisation de la courbe de fréquence cumulée (SSD, distribution de sensibilité des espèces) pour déterminer une valeur de référence sans effet.

Use of the cumulative frequency curve (SSD, Species Sensibility Distribution) to determine a no effect reference value.

classique (normale, log-normale, log-logistique ${ }^{2}$, etc.) à la distribution statistique d'un critère d'effet donné $\left(\mathrm{CE}_{x}\right.$ ou $\mathrm{DDE}_{x}$, soit respectivement Concentration et Débit de Dose ayant un Effet de $x \%$ pour des individus exposés par rapport à un témoin). En fixant le seuil de protection à (1-p) \% d'espèces constituant l'écosystème, la valeur de référence sans effet est lue directement sur la courbe : elle correspond à l'abscisse associée à l'ordonnée $p \%$ (Fig. 3). Usuellement, le pourcentage d'espèces à protéger est fixé à $95 \%$, la valeur de référence sans effet associée étant alors dénommée $\mathrm{HC}_{5}$ (concentration dangereuse - Hazardous Concentration - pour $5 \%$ des espèces). Cette méthode, qui permet de tracer l'utilisation de chaque donnée d'écotoxicité, est a priori plus scientifique que la précédente, dans le sens où elle ne fait pas appel à la seule plus petite valeur d'écotoxicité, et lui est à ce titre préférée lorsque elle est employable. Toutefois,

\footnotetext{
2 Loi log-logistique : cette loi permet de passer outre les inconvénients de la loi normale dont la fonction de répartition n'a pas d'expression analytique. De paramètres $\alpha$ et $\theta$, elle a pour fonction de densité $f(t)=\alpha \theta \frac{(\alpha t)^{\theta}}{\alpha t\left[1+(\alpha t)^{\theta}\right]^{2}}$.
} 
TABLEAU II

Principaux critères susceptibles de discriminer les méthodes de caractérisation du risque environnemental.

Main potential distinction criteria between existing environmental risk assessment methods.

\begin{tabular}{ll}
\hline Critères & Options possibles \\
\hline Grandeur utilisée pour la comparaison exposition-effet & Concentration, dose (débit de dose), indice de risque \\
$\begin{array}{l}\text { Type de valeur utilisée pour la comparaison } \\
\text { exposition-effet }\end{array}$ & Valeur limite prédéfinie ou définie pour l'étude... \\
Méthode de comparaison & Déterministe, semi-probabiliste, probabiliste \\
Méthodes d'extrapolation si nécessaire & $\begin{array}{l}\text { Facteurs d'extrapolation, distributions probabilistes, } \\
\text { modèles empiriques... }\end{array}$ \\
Évaluation des incertitudes & Stratégie définie ou non \\
Prise en compte du bruit de fond & Incrémentation / estimation globale \\
\hline
\end{tabular}

les limites de toute approche statistique (adéquation de la loi de distribution, sensibilité au nombre et à la qualité des données) en contraignent l'utilisation et la validité. Une discussion approfondie sur ce point peut être consultée dans (IRSN, 2006).

Cette dernière approche, dite des SSD, a été mise en œuvre par l'IRSN (Garnier-Laplace et al., 2006) dans le cadre du projet européen ERICA (20042007 ; ERICA, 2007). Les données d'écotoxicité utilisées pour établir les SSD correspondantes sont cependant toutes issues d'irradiation gamma externe. Leur application à l'ensemble des conditions d'exposition (interne, externe) et de rayonnements (gamma, alpha, beta) nécessite l'application de facteurs de sécurité, afin de rendre compte notamment de la différence d'efficacité biologique des rayonnements selon leur nature.

Une seconde utilisation est faite des courbes de fréquence cumulée, dont la lecture à partir de l'axe des abscisses (données d'exposition) conduit à déterminer par l'ordonnée associée la PAF (Potentially Affected Fraction), fraction potentiellement affectée des espèces.

\subsection{Caractérisation du risque}

Disposant des résultats des phases d'analyse des effets et de l'exposition, il s'agit ensuite de caractériser le risque pour l'environnement, plusieurs critères (Tab. II) pouvant alors à nouveau discriminer les méthodes existantes d'évaluation du risque écologique ( $c f$. Sect.1.2).

L'élargissement aux radionucléides du champ d'application de cette méthode aujourd'hui classique d'évaluation du risque pour l'environnement a permis de 
faire un premier pas vers l'harmonisation entre domaine radiologique et domaine chimique; elle n'autorise cependant toujours pas la caractérisation de l'importance relative de chaque contaminant dans l'impact écologique global potentiel d'un mélange de polluants de différentes natures, comme par exemple les effluents liquides rejetés en fonctionnement normal par les centres nucléaires de production d'électricité.

\section{Méthode d'évaluation comparative du risque radiologique et chimique pour l'environnement}

Inspirée des méthodes mises en œuvre pour l'évaluation des impacts dans le cadre de l'analyse du cycle de vie d'une substance (LCIA, Life Cycle Impact Assessment), l'approche développée pour les substances chimiques (Payet et Jolliet, 2003) est en cours d'adaptation pour les radionucléides, en vue de l'évaluation comparative du risque chronique pour les écosystèmes aquatiques lié au mélange de substances chimiques et radioactives.

\subsection{Théorie générale de l'évaluation comparative du risque écologique}

Il s'agit de calculer un facteur d'effet relatif à l'impact écotoxicologique, commun à tous les types de stresseurs : cet indicateur est le changement de la fraction d'espèces potentiellement affectées (ou $\triangle P A F$ ) par le changement de la concentration d'exposition, et ce pour chacun des stresseurs $i$ considérés, soit le ratio $\triangle P A F_{i} / \Delta C_{i}$ (Pennington et al., 2006). Pour calculer cet indicateur, il est possible d'utiliser non plus la concentration dangereuse pour $5 \%$ des espèces $\left(\mathrm{HC}_{5}\right)$ déterminée à partir des concentrations ayant un effet sur $10 \%$ des individus exposés $\left(\mathrm{CE}_{10}\right)$, mais plutôt la concentration dangereuse pour $50 \%$ des espèces $\left(\mathrm{HC}_{50}\right)$, évaluée à partir des concentrations ayant un effet sur $50 \%$ des individus exposés $\left(\mathrm{CE}_{50}\right)$. En effet, l'incertitude sur la $\mathrm{HC}_{50}$, médiane de la distribution, est plus faible que celle sur le cinquième percentile $\left(\mathrm{HC}_{5}\right)$, et cette médiane peut en outre être calculée sans avoir à formuler d'hypothèse quant à la forme de la distribution de la SSD (Payet et Jolliet, 2003). De même, l'utilisation des $\mathrm{CE}_{50}$, dont la détermination est plus robuste que celle des $\mathrm{CE}_{10}$, contribue à réduire l'incertitude sur l'indicateur global d'effet.

Pour une substance $i$ isolée dont la SSD suit une loi log-logistique, le facteur d'effet se calcule alors selon l'équation (5), sous l'hypothèse d'un gradient linéaire entre l'origine et la valeur de référence $\mathrm{HC}_{50}$ (Fig. 4). Pour un mélange $m s$ de substances, sous l'hypothèse de l'addition des concentrations (mélange de substances de mode d'action similaire) comme sous celle de l'addition des 


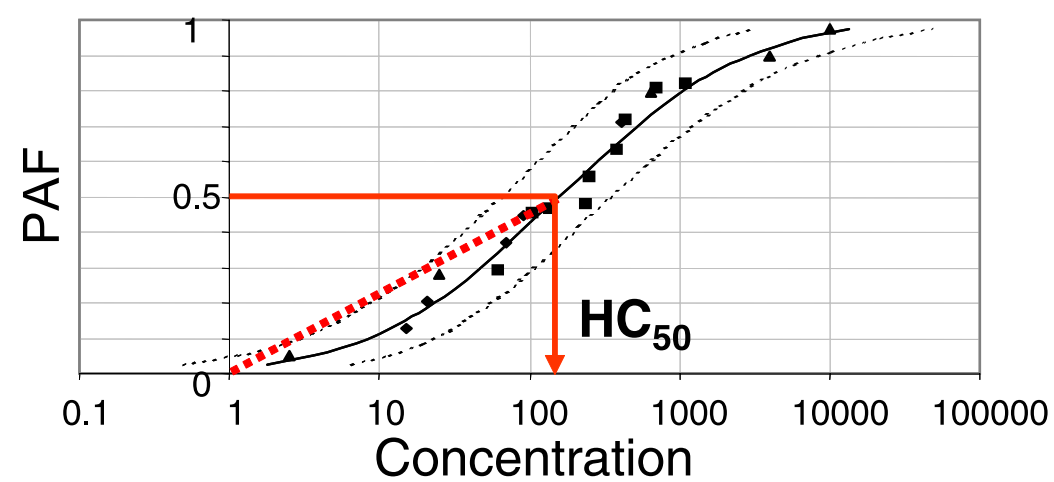

Figure 4 - Détermination de la variation de PAF (fraction d'espèces potentiellement affectée) en fonction de la variation de concentration d'exposition (sous l'hypothèse d'un gradient linéaire de 0 à $50 \%$ de PAF).

Determination of the PAF (Potentially Affected Fraction) variation according to the exposure concentration variation (considering a linear relation between 0 and 50\% of PAF).

réponses (mélange de substances de mode d'action indépendant), le facteur d'effet est obtenu d'après l'équation (6) (Pennington et al., 2004).

substance isolée : $\triangle P A F_{i}=\Delta C_{i} \times \frac{0,5}{H C_{50}}$,

mélange de substances : $\triangle P A F_{m s}=0,5 \times \sum_{i} \frac{0,5}{H C_{50_{i}}}$.

\subsection{Adaptation aux radionucléides}

Parmi les propriétés qui différencient les radionucléides des substances chimiques classiques, deux sont à prendre en compte dans l'extension de la méthode proposée pour le second type de contaminant au premier : l'expression des effets en termes de dose absorbée ou de débit de dose, et les différentes voies d'exposition possibles de l'organisme cible, à savoir dans les écosystèmes aquatiques l'irradiation externe, à la fois à l'eau et au sédiment, et la contamination.

L'analyse des effets selon la méthodologie préconisée au précédent paragraphe conduit à la détermination des $\mathrm{DDE}_{50}$, débits de dose ayant un effet sur $50 \%$ des individus exposés (en reprenant les données utilisées pour déterminer les débits de dose ayant un effet sur $10 \%$ des individus exposés, ou DDE D $_{10}$; Garnier-Laplace et al., 2006), débits de dose dont les unités ne sont pas compatibles avec celles employées pour les substances chimiques, les $\mathrm{CE}_{50}$ étant des concentrations. 
La moyenne géométrique de ces $\mathrm{DDE}_{50}$ donne le $\mathrm{HDR}_{50}{ }^{3}$ (débit de dose dangereux pour $50 \%$ des espèces), qui est ensuite converti par rétro-calcul en $\mathrm{CE}_{50}$ pour chacun des compartiments d'intérêt, biotique (organisme cible) et abiotique (eau et sédiment), selon les équations (8) et (9). $\mathrm{La} \mathrm{HC}_{50}$ peut alors être obtenue en faisant la moyenne géométrique de ces $\mathrm{CE}_{50}$

$\mathrm{CE}_{50}($ organisme,eau $)=$

$\frac{H D R_{50}}{F O(\text { organisme,eau }) \times D C C_{\text {eau }}(\text { rn,organisme })+B C F(\text { rn,organisme }) \times D C C_{\text {int }}(\text { rn,organisme })},(8)$

$\mathrm{CE}_{50}($ rn,organisme,sédiment $)=\frac{H D R_{50}}{F O(\text { organisme, sédiment }) \times D C C_{\text {sed }}(\text { rn,organisme })}$.

\subsection{Application aux effluents liquides de routine d'un CNPE}

À titre d'illustration de la méthode, les résultats obtenus sur les données réelles $(E D F, 2005)$ de rejets liquides chimiques et radioactifs d'un centre nucléaire de production d'électricité $(2 \times 1300 \mathrm{MW})$ sont présentés de façon très succincte, les détails de l'étude étant décrit par ailleurs (Garnier-Laplace et al., 2007). Les substances concernées sont celles usuellement présentes dans ces effluents, à savoir :

- cuivre, zinc, acide borique, ammonium, chlorure de sodium, détergent, morpholine et lithine pour les substances chimiques ;

- ${ }^{3} \mathrm{H},{ }^{14} \mathrm{C},{ }^{54} \mathrm{Mn},{ }^{58} \mathrm{Co},{ }^{60} \mathrm{Co},{ }^{63} \mathrm{Ni},{ }^{110 \mathrm{~m}} \mathrm{Ag},{ }^{123 \mathrm{~m}} \mathrm{Te},{ }^{124} \mathrm{Sb},{ }^{125} \mathrm{Sb},{ }^{131} \mathrm{I},{ }^{134} \mathrm{Cs}$ et ${ }^{137} \mathrm{Cs}$ pour les radionucléides.

Les conditions de rejet sont représentatives d'une situation annuelle moyenne, avec un débit du cours d'eau récepteur fixé à $1040 \mathrm{~m}^{3} \mathrm{~s}^{-1}$.

L'application de la méthode proposée a permis de classer les différentes substances étudiées en fonction de leurs impacts individuels relatifs exprimés en $\triangle P A F$ (Fig. 5). L'impact total du rejet demeure très faible, et la contribution majeure provient très largement des substances chimiques, l'impact des radionucléides étant inférieur d'environ 6 ordres de grandeur. Par ailleurs, pour les radionucléides concernés, l'impact radiotoxique est supérieur à leur impact chimiotoxique.

\section{Conclusions}

Une première étape méthodologique a été franchie en proposant une méthode d'évaluation du risque écologique associé à la présence ou au rejet de

\footnotetext{
$3 \mathrm{La} \mathrm{HC}_{50}$ ou le $\mathrm{HDR}_{50}$ peut être calculé(e) comme la moyenne, la médiane ou la moyenne géométrique des $\mathrm{CE}_{50}$ ou des $\mathrm{DDE}_{50}$. La distribution des $\mathrm{CE}_{50}$ et les $\mathrm{DDE}_{50}$ suivant généralement une loi log-normale, l'estimateur statistique le plus adapté aux besoins de la méthode, i.e. le moins sensible à la loi de distribution, est alors la moyenne géométrique (Payet, 2004).
} 


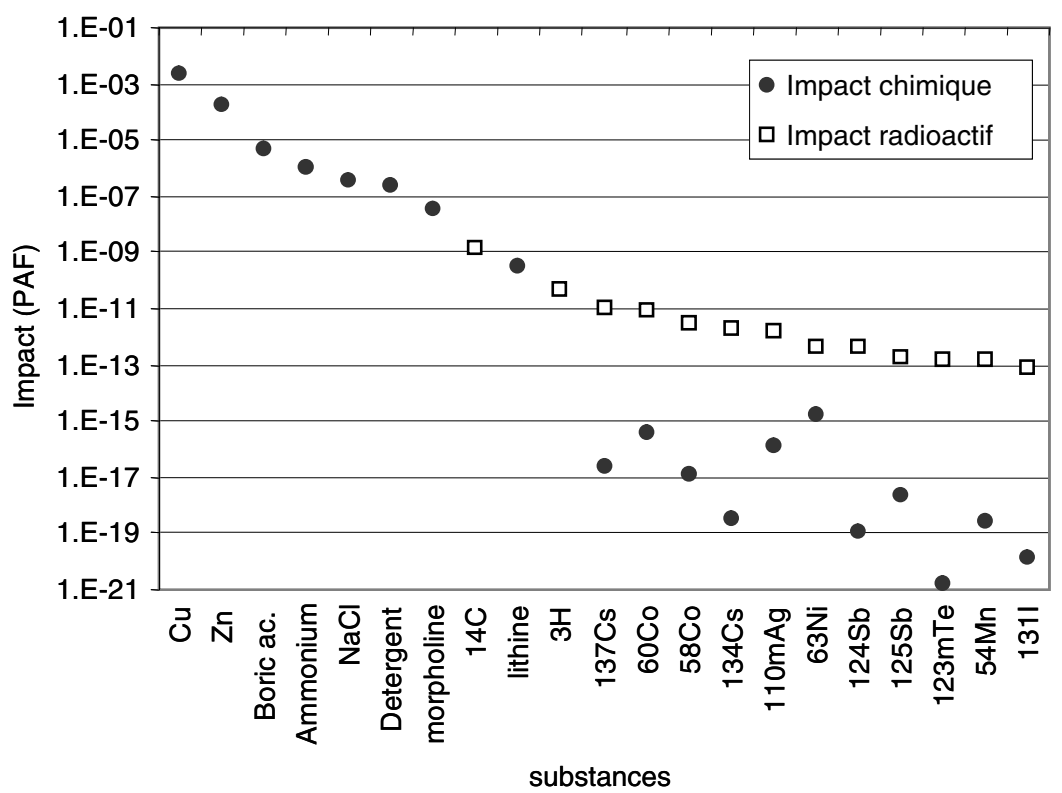

Figure 5-Impact comparé des substances chimiques et radioactives (PAF : fraction d'espèces potentiellement affectée).

Comparative impact of chemical and radioactive substances (PAF: Potentially Affected Fraction).

radionucléides dans l'environnement cohérente avec celle classiquement utilisée pour les substances chimiques. Mais ce n'est pas encore suffisant dans le contexte de pollution multiple qui est celui auquel sont aujourd'hui confrontés les écosystèmes, l'approche classique, mono-stresseur, étant en outre très conservative.

Devant les besoins en termes d'évaluation comparative des risques pour l'environnement, les développements méthodologiques en cours, inspirés des méthodes appliquées en analyse du cycle de vie des produits, ont été présentés, avec les spécificités liées aux propriétés caractéristiques des radionucléides. Une première série de résultats a été obtenue par application de la méthode proposée au cas des effluents liquides radioactifs et chimiques rejetés en fonctionnement normal par un centre nucléaire de production d'électricité. La faisabilité d'une évaluation comparative du risque radiologique et du risque chimique est donc prouvée, le cas d'étude présenté ayant permis d'établir d'une part pour les radionucléides à la fois leur impact radiotoxicologique et chimiotoxicologique, 
et d'autre part de comparer l'impact radiotoxicologique des substances radioactives avec l'impact chimiotoxicologique des substances chimiques. Toutefois, des développements complémentaires sont nécessaires, pour rendre la méthode plus robuste, y introduire la prise en compte des incertitudes et hiérarchiser l'importance relative des différents paramètres des modèles employés, afin d'identifier les points critiques nécessitant un approfondissement dans la définition ou la détermination des paramètres associées.

Remerciements. Les auteurs remercient Jérôme Payet et Olivier Jolliet pour les fructueuses discussions qui ont conduit à la proposition de la méthode comparative d'évaluation des risques radiologique et chimiotoxique pour l'environnement. Ce travail a par ailleurs bénéficié, pour la partie générale relative à l'évaluation du risque pour l'environnement associé à la présence de radionucléides, du support financier d'EDF (Groupe de gestion de projetsenvironnement) et de la Commission européenne (ERICA).

\section{RÉFÉRENCES}

Beaugelin-Seiller K., Jasserand F., Garnier-Laplace J., Gariel J.C. (2006) Modelling the radiological dose in non-human species: principles, computerization and application, Health Phys. 90, 485493.

EC (2003) Technical Guidance Document in spport of Commission Directive 93/67/EEC on risk assessment for new notified substances and Commission Regulation (EC) $n^{\circ} 1488 / 94$ on risk assessment of existing substances, Directive 98/8/EC of the European Parliament and of the Council concerning the placing of biocidal products on the market, Part II, Office for Official Publication of the European Communities, Luxembourg.

EDF (2005) Nucléaire et environnement 2005, EDF Division Production Nucléaire, St Denis (France).

ERICA (2007) D-ERICA: An INTEGRATED APPROACH to the assessment and management of environmental risks from ionising radiation. ERICA Project, European Commission, contract $\mathrm{n}^{\circ}$ FI6R-CT-2004-508847.

Garnier-Laplace J., Della-Vedova C., Gilbin R., Copplestone D., Ciffroy P. (2006) First Derivation of Predicted-No-Effect Values for Freshwater and Terrestrial Ecosystems Exposed to Radioactive Substances, Environ. Sci. Techn. 40, 6498-6505.

Garnier-Laplace J., Beaugelin-Seiller K., Payet J., Gilbin R. (2007) A screening level risk assessment and ranking method for chemical and radioactive mixtures released in freshwaters by nuclear facilities under normal operating conditions, SETAC-Europe, 20-24 mai, Porto (Portugal).

Gonze M.A., Mourlon C., Chen T., Garcia-Sanchez L., Le Dizes S. (2004) SYMBIOSE: A Modeling and Simulation Platform for Environmental Pollutant Risk Assessment Studies, Eurosafe 2004, 8-9 novembre, Berlin (Allemagne).

ICPR Publication 60 (1991) 1990 Recommendations of the International Commission on Radiological Protection, Ann. ICRP 21.

IRSN (2006) Radioprotection de l'environnement / Synthèse et perspectives, édition du $1^{\mathrm{er}}$ juillet 2006, ISR IRSN-2005/56-FR, Collection Doctrine et synthèse, IRSN, Clamart (France).

Payet J. (2004) Assessing toxic impacts on aquatic ecosystems in Life Cycle Assessment (LCA). Thèse $n^{\circ}$ 3112, Ecole Polytechnique Fédérale de Lausanne, Institut des Sciences et Technologies de l'Environnement, section Sciences et Ingénierie de l'Environnement. 
Payet J., Jolliet O. (2003) Comparative assessment of the toxic impact of metals on aquatic ecosystems: the AMI method, Dans Life-cycle assessment of metals: Issues and Research Directions (Dubreuil Ed.), SETAC, Pensacola, FL (USA).

Pennington D.W., Payet J., Hauschild M. (2004) Aquatic ecotoxicological indicators in life-cycle assessment, Environ. Toxicol. Chem. 23, 1796-1807.

Pennington D.W., Margni M., Payet J., Jolliet O. (2006) Risk and Regulatory Hazard-Based Toxicological Effect Indicators in Life-Cycle Assessment (LCA), Hum. Ecol. Risk Assess. 12, 450-475.

Posthuma L., Traas T., Suterll G. (2002) General Introduction and history of SSDs, Dans Species Sensitivity Distributions in ecotoxicology (Suterll Ed.), Lewis, Boca Raton, London, New York, Washington DC, pp. 3-36.

Suter G., Efroymson R., Sample B., Jones D. (2000) Ecological risk assessment for contaminated sites, Lewis, Boca Raton, London, New York, Washington DC. 